\title{
Publisher's Note: Generic patterns in the evolution of urban water networks: Evidence from a large Asian city [Phys. Rev. E 95, 032312 (2017)]
}

Elisabeth Krueger, Christopher Klinkhamer, Christian Urich, Xianyuan Zhan, and P. Suresh C. Rao

(Received 7 March 2019; published 13 March 2019)

DOI: 10.1103/PhysRevE.99.039903

This paper was published online on 9 March 2017 with an error in Eq. (1b) and surrounding text and in the caption to Fig. 6. The caption of Fig. 6 should read as

"WDN subnets along a gradient of breaking points between the power laws of trunk and tail ( $k_{\text {break }}$ outliers from panel (d) in Fig. 5): (a) DZ11: $k_{\text {break }}=5, p(k)_{\text {trunk }}=0.40 k^{-2.49}$ and $p(k)_{\text {tail }}=0.09 k^{-1.47}, N=2425$ (dual-mapped nodes); (b) DZ10: $k_{\text {break }}=8, p(k)_{\text {trunk }}=1.46 k^{-2.80}$ and $p(k)_{\text {tail }}=0.26 k^{-1.88}, N=3179$; (c) DZ01: $k_{\text {break }}=10, p(k)_{\text {trunk }}=1.17 k^{-2.37}$ and $p(k)_{\text {tail }}=0.138 k^{-1.686}, N=1497$; (d) DZ32: in this subnet power-law distributions of trunk and tail converge as the breaking point between the two power laws increases $\left(k_{\text {break }}=20\right)$, and $p(k)=1.07 k^{-2.27}(N=2271)$."

On page 2, left-hand column, the text above Eqs. (1a) and (1b) should read as "The node degree distributions (NDD) for both types of water networks can be approximated by a Pareto power-law distribution [Eq. (1a); large, mature networks],

$$
p(k)=a k^{-\gamma}
$$

for $k \geqslant 2$, or a double Pareto power-law distribution [Eq. (1b); small, immature networks], described by a two-piece function in the form

$$
p(k)_{\text {trunk }}=a k^{-\gamma_{\text {trunk }}}, \quad p(k)_{\text {tail }}=b k^{-\gamma_{\text {tail }}},
$$

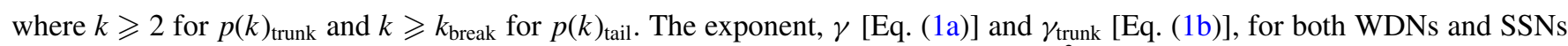
converges above a threshold of network size, measured as dual-mapped nodes $N>10^{2}$."

The paper has been corrected as of 25 February 2019. The caption and text are incorrect in the printed version of the journal. 